\title{
Les formes élémentaires d'agir performatif. Un essai de typologie sociologique
}

The Elementary forms of performative Acts

Las formas elementales de expresarse en la actuación. Un ensayo de tipología sociológica

\author{
Werner Binder, Sophie Schinko, Werner Binder et Francis Le Maître
}

Numéro 51, automne 2011

Théâtralité et société : positions de la sociologie

URI : https://id.erudit.org/iderudit/1014998ar

DOI : https://doi.org/10.7202/1014998ar

Aller au sommaire du numéro

Éditeur(s)

Athéna éditions

ISSN

0831-1048 (imprimé)

1923-5771 (numérique)

Découvrir la revue

Citer cet article

Binder, W., Schinko, S., Binder, W. \& Le Maître, F. (2011). Les formes

élémentaires d'agir performatif. Un essai de typologie sociologique. Cahiers de recherche sociologique, (51), 75-96. https://doi.org/10.7202/1014998ar

\section{Résumé de l'article}

L'article étudie l'emploi du concept de performance au sein de la théorie sociologique contemporaine et développe pour lui-même une typologie de l'action performative en trois parties. Primo, les performances ritualisées se concentrent sur la manière dont ses participants s'y conforment. Secundo, les performances théâtralisées sont caractérisées par la fictionalité et introduisent ainsi une distinction entre acteurs et auditoires. Tertio, les performances sociales sont aussi distinctes des auditoires, mais ont ceci de particulier qu'elles doivent apparaître authentiques si elles veulent avoir un certain succès. Ces trois formes élémentaires constituent chacune un ensemble complexe ayant des règles propres. Ceci dit, des confluences et des interactions existent, telles les fois où les drames théâtraux empruntent aux événement historiques ou lorsque les acteurs sociaux s'inspirent de performances fictionnelles afin de recréer de la ritualité, et donc une certaine adhésion de la part des auditoires. À terme, il doit devenir clair que toutes ces formes de performance sont fortement entrelacées dans les faits, c'est-à-dire qu'elles puisent à ce même réservoir qu'est l'imaginaire social. 


\section{Les formes élémentaires d'agir performatif. Un essai de typologie sociologique}

WERNER BINDER

\section{Introduction}

Sans nul doute, le vocabulaire du théâtre a laissé des traces profondes dans la terminologie et la manière même dont se pense la discipline sociologique. Non seulement l'expression "acteur» a été introduite pour désigner celui qui agit, mais aussi l'ensemble des attentes liées à la position sociale d'une personne se trouve souvent qualifié de «rôles». Et c'est sans compter sur tous ces sociologues qui empruntent explicitement des concepts théâtraux comme «mise en scène ${ }^{1}$ » pour décrire des processus sociaux plus complexes. Donnant suite à ces approches classiques et modernes, il s'agira ici de répondre à la question de savoir si la métaphore théâtrale peut encore faire fructifier la théorie sociologique ainsi que l'auto-description de la société ou bien, au contraire, si elle reste trop marginale et faible heuristiquement. Aussi, afin de préciser cette idée d'une théâtralité appliquée à la sociologie, il faudra incorporer un autre concept à l'analyse, à savoir celui de rituel. C'est que l'emploi de ce concept est aussi ancien que celui de la métaphore du théâtre et qu'il doit rendre compte de ce que, très justement, les rituels jouent un

1. Voir Erving Goffman, La mise en scène de la vie quotidienne, Paris, Minuit, 1992 et Jeffrey C. Alexander, «Cultural Pragmatics: Social Performance between Ritual and Strategy», dans Jeffrey C. Alexander, Bernhard Giesen et Jason L. Mast (dir.), Social Performance. Symbolic Action, Cultural Pragmatics, and Ritual, Cambridge, Cambridge University Press, 2006, p. 29-90. 
rôle central non seulement dans les sociétés archaïques, mais également dans les sociétés modernes ${ }^{2}$.

Entre la pratique d'un rite et la représentation d'une pièce de théâtre, il y a ainsi des différences considérables comme autant des ressemblances remarquables, et c'est davantage sur ces dernières que nous voudrions insister. Celles-ci donnent à penser que la ritualité et la théâtralité se retrouvent à l'intérieur d'un concept principal de "performance» qui lui-même se différencie tout à fait des autres formes d'agir. Au cours des dernières années, quelques recueils marquants sur la performativité ont été publiés et c'est à partir de ceux-ci que les réflexions qui s'ensuivent voudront s'orienter ${ }^{3}$. L'objectif de ce texte est ainsi de développer une typologie de l'agir performatif qui, bien que théorique, se prête à une analyse plus empirique de phénomènes sociaux. Au-delà, nous aimerions démontrer le rôle que jouent les formes élémentaires de performance dans les processus sociaux complexes de même que la manière dont elles interagissent fortement avec l'imaginaire d'une société.

Aussi bien les performances rituelles que théâtrales sont dévaluées comme des formes pour ainsi dire impropres par de nombreuses théories contemporaines de l'action et, quelques exceptions essentielles mises à part, laissées de côté. Pour prendre cet important exemple, Jürgen Habermas s'est efforcé, dans sa Théorie de l'agir communicationnel, de mettre en langage le concept durkheimien de rituel en transformant «la force de fascination du sacré» en quelque chose comme «la force contraignante des prétentions à la validité critiquable ${ }^{4} »$. Par cette distorsion rationaliste, Habermas aliène toutefois les conceptions relevant plus proprement de la sociologie culturelle dans l'œuvre tardive de Durkheim. Ses efforts iconoclastes défigurent ainsi le rite jusqu'au méconnaissable en le remplaçant par un concept d'agir communicationnel qu'il oppose à l'agir stratégique motivé par intérêt. Certes, Habermas développe l'agir dramaturgique comme troisième type d'agir, mais il le banalise en même temps en lui attribuant surtout une fonction esthétique et expressive dans le domaine artistique ${ }^{5}$. Eu égard à la supériorité de l'action communicative et stratégique, dont la dualité pour Habermas

2. Émile Durkheim, Les formes élémentaires de la vie religieuse. Le système totémique en Australie, Paris, Livre de Poche, 1991; Jeffrey C. Alexander, «Watergate as Democratic Ritual», dansThe Meanings of Social Life. A Cultural Sociology, Oxford, Oxford University Press, 2003, p. 155-177 et Ronald L. Grimes et al. (dir.), Ritual, Media, and Conflict, Oxford, Oxford University Press, 2011.

3. Jeffrey C. Alexander, Bernhard Giesen et Jason L. Mast (dir.), op. cit.; Christoph Wulf, Michael Göhlich et Jörg Zirfas (dir..), Grundlagen des Performativen. Eine Einführung in die Zusammenhänge von Sprache, Macht und Handeln, Weinheim, Juventa, 2001.

4. Jürgen Habermas, Théorie de l'agir communicationnel. Tome 1. Rationalité de l'agir et rationalisation de la société, Paris, Fayard, 1987, p. 88.

5. Ibid., p. 106-111. 
constitue la totalité de la sociétéé, l'action dramaturgique se retrouve alors à être «parasitaire ${ }^{7}$ » et sans grandes conséquences en comparaison. La thèse ici défendue est que l'action dramaturgique ou bien performative est indispensable pour une compréhension des sociétés et du public modernes et qu'en ce sens elle ne peut être traitée de manière raisonnable dans un cadre tel celui d'Habermas. La performativité n'est pas un élément décoratif et donc superflu de l'action, mais est au contraire d'une importance existentielle en ce qui concerne les questions sociétales sur la guerre et la paix, la vie et la mort, le vrai et le faux, etc. Ces formes d'action «impropres» développent une capacité ou un pouvoir sociétal qui est toujours largement sous-estimé par beaucoup de théoriciens.

\section{Performativité - mimesis - résonance}

Au cours des dernières années, le concept et l'idée de performativité ont connu une attention de plus en plus soutenue au sein des sciences humaines ${ }^{8}$. Cependant, son emploi reste très hétérogène puisqu'il lui manque jusqu'à aujourd'hui une définition applicable ou pour le moins un tant soit peu consensuelle. Cela a des raisons historiques importantes: l'emploi des mots «performatif», "performance» et "performativité» se rapporte, d'une part, à l'empreinte propre des linguistes et des philosophes, mais renvoie aussi, d'autre part, à l'appropriation universitaire du monde du théâtre. La définition et la typologie proposées dans cette première partie ont l'intention de clarifier cette confusion linguistique et ainsi permettre l'élaboration graduelle d'une distinction importante qu'il faudra faire entre performance rituelle, théâtrale et sociale.

Il importe de spécifier le concept de performativité afin de le distinguer clairement des autres formes et aspects de l'agir. C'est qu'il serait tout à fait plausible de penser que tout agir, considéré comme l'accomplissement d'une action, serait nécessairement performatif. Une telle interprétation du concept de performance correspond entre autres à celle de Noam Chomsky9. «Performance» dans ce cas signifie la sélection et l'actualisation d'un acte de langage devant une multitude de possibilités révélées par la compétence du locuteur. Cela s'explique particulièrement bien par l'exemple du multilinguisme: si un locuteur est bilingue ou sait parler un dialecte, il possède

6. Sur la distinction entre «système» et «Lebenswelt», voir Jürgen Habermas, Théorie de l'agir communicationnel. Tome 2. Pour critique de la raison fonctionnaliste, Paris, Fayard, 1987, p. 125-216.

7. Jürgen Habermas, Théorie de l'agir communicationnel. Tome 1, op. cit., p. 106.

8. Par exemple Judith Butler, Excitable Speech. A Politics of the Performative, New York, Routledge, 1997; Jeffrey C. Alexander et al. (dir..), Social Performance, op. cit.; Erika Fischer-Lichte, The Transformative Power of Performance. A New Aesthetics, Londres, Routledge, 2008.

9. Noam Chomsky, Language and Mind, Cambridge, Cambridge University Press, 2007. 
alors la compétence de changer les codes linguistiques de ses propres performances selon la situation. Un tel raisonnement se laisse tout à fait transposer dans une théorie générale de l'action et peut même être franchement utile en sociologie culturelle - comme le démontre l'exemple de la culture en tant que «toolkit» chez Swidler ${ }^{10}$. Ainsi, de la même manière que Ferdinand de Saussure fait la différence entre la parole et la langue, il faut comprendre que la langue ne se rapporte pas à la compétence du locuteur individuel, mais qu'elle fixe l'horizon d'un langage ${ }^{11}$. L'emploi du concept de performance visant ici la sélection ou l'actualisation d'un acte possible est l'interprétation de ce même concept non seulement la plus générale, mais aussi la plus banale.

John Austin n'avait pas en tête cet usage général de la notion quand il a abordé lesdits «performatifs» dans son étude pionnière How to do things with words ${ }^{2}$ - dans laquelle justement il dépasse le fait banal que tout langage est également agir. Austin réserve le terme "performatif» uniquement pour la désignation des actes de langage créant des faits sociaux et des obligations par leur simple énonciation, comme par exemple la promesse et l'excuse. Pour expliquer ce mystérieux pouvoir des mots, Austin instaure une distinction entre différents aspects de l'agir langagier. L'acte locutoire définit la chose d'un énoncé tandis que l'acte perlocutoire désigne l'intention du locuteur (au sens de l'effet prévu sur les auditeurs). Par rapport aux précédents, lesdits "performatifs» se caractérisent par la domination d'une troisième forme de l'acte. En effet, l'acte illocutoire lie le locuteur à une obligation endossée par l'acte de langage même. En disant «Promis!» on s'oblige à la mise en œuvre et la matérialisation de cette promesse. La théorie d'Austin sur les actes de langage et son développement par John Searle ont eu de la sorte une influence extraordinaire - par exemple jusque dans la théorie de l'agir communicationnel qui traduit la distinction entre l'acte illocutoire et l'acte perlocutoire en l'opposition entre l'agir communicatif et stratégique ${ }^{13}$. Aussi le concept de Bourdieu du «pouvoir symbolique ${ }^{14} »$ provient de la force d'illocution des expressions linguistiques d'Austin.

Toujours chez les philosophes, Jacques Derrida n'est pas le seul à avoir critiqué la théorie du langage d'Austin. Il n'empêche qu'il importe de s'at-

10. Ann Swidler, «Culture in Action. Symbols and Strategies», American Sociological Review, vol. 51, $\mathrm{n}^{\circ}$ 2, 1986, p. 273-286. Swidler pose en effet que les acteurs ont à leur disposition un répertoire culturel limité au sein duquel ils peuvent choisir librement selon des considérations stratégiques. Voir aussi la critique de Stephen Vaisey pour qui le «toolkit», comme modèle de répertoire, néglige la culture vue comme une variable motivationnelle, causale et autonome, «Motivation and Justification. Toward a Dual Process Theory of Culture in Action», American Journal of Sociology, vol. 114, nº 6, 2009, p. 1675-1715 (p. 1678-1682 et p. 1704-1708).

11. Ferdinand de Saussure, Cours de linguistique générale, Paris, Payot, 1979.

12. John L. Austin, Quand dire, c'est faire, Paris, Seuil, 1970.

13. Jürgen Habermas, Théorie de l'agir communicationnel. Tome 1, op. cit.

14. Pierre Bourdieu, Ce que parler veut dire. L'économie des échanges linguistiques, Paris, Fayard, 1982, p. 97-161. 
tarder à sa théorie déconstructionniste puisqu'elle montre une voie vers la typologie qui sera proposée ici ${ }^{15}$. Restant dans la tradition de Saussure, Derrida montre que l'acte de langage ne déploie pas sa force illocutoire dans un événement singulier, mais seulement par une répétition et une citation renvoyant à un savoir social plus largement partagé. La signification de "performatifs» est dès lors toujours canonisée, ce qui l'approche des performances rituelles: «le "rite" n'est pas une éventualité, c'est, en tant qu'itérabilité un trait structurel de toute marque ${ }^{16} »$. Nous verrons plus loin que les rituels classiques possèdent également une teneur illocutoire qui est responsable de leur effectivité sociale. Derrida expose d'une façon convaincante que les «performatifs» peuvent seulement fonctionner en tant que citations d'un texte culturel. Mais il faut encore en différencier le citer «impropre» venant du théâtre, le fait de réciter. Pour Derrida, cette forme «impropre» de citer cause des problèmes insolubles à Austin, c'est pourquoi ce dernier l'exclut de son analyse des performatifs:

[...] une énonciation performative sera creuse ou vide d'une façon particulière si, par exemple, elle est formulée par un acteur sur la scène, ou introduite dans un poème, ou émise dans un soliloque. [...] Tout cela, nous l'excluons donc de notre étude. Nos énonciations performatives, heureuses ou non, doivent être entendues comme prononcées dans des circonstances ordinaires ${ }^{17}$.

Le compte rendu d'une excuse ou la représentation théâtrale d'une promesse ne représentent pour Austin nullement un acte de langage performatif. Par conséquent, il lui manque la teneur illocutoire et l'effectivité sociale. Citer devient ici le parasite de la parole propre. Selon Derrida, Austin cache par son omission le fait que la force illocutoire d'un acte performatif ne peut pas tenir seulement à son acte de langage, mais qu'elle renvoie au contexte plus précis de l'acte.

L'agir langagier ne peut pas être strictement séparé de son contexte car celui-ci est toujours indiqué par l'acte de langage même. L'utilisation impropre des expressions linguistiques dans la citation et la récitation correspond à la fictionalité des performances théâtrales. Les éléments constitutifs du rituel, la répétition et la citation, jouent un rôle dans ces performances. Ils apparaissent cependant dans une configuration spécifique qui marque en même temps une différence par rapport au rituel. La fictionalité d'un acte dépend, ainsi que la teneur illocutoire, de la contextualisation qui reste toujours précaire et qui, par conséquence, peut échouer. Par exemple, un mariage sur une scène ne signifie pas pour les participants et les auditeurs la même chose

15. Jacques Derrida, Marges de la philosophie, Paris, Éditions de Minuit, 1972.

16. Ibid., p. 385.

17. John L. Austin cité dans Jacques Derrida, Marges de la philosophie, op. cit., p. 386-387. 
qu'un mariage au bureau de l'État civil. Une dernière critique derridienne des travaux d'Austin vise ainsi à la possibilité inhérente de l'échec de tous les actes performatifs ${ }^{18}$. Les conditions de félicité ou de réussite servent plutôt à l'exclusion de l'échec qu'à son intégration théorique. Une promesse ou une excuse peuvent très bien échouer quand la performance n'arrive pas à convaincre le destinataire de la sincère intention de son auteur. Pour Austin, la force illocutoire d'une expression dépend de l'intention du locuteur, mais pas de la compréhension de l'auditeur. La réduction de l'effet performatif sur l'intention du locuteur est de ce fait trop simpliste. Contrairement à Austin, une théorie sociologique de la performance doit considérer que l'effectivité sociale des intentions repose sur les attributions des autres acteurs et que celles-ci dépendent à leur tour de la performance de l'acte de langage. La sincérité de l'intention du locuteur reste alors dépendante de la crédibilité performativement obtenue par l'acte. Autrement dit, la force illocutoire d'un acte de langage ne se dissocie plus de son succès perlocutoire, c'est-à-dire de son effet tel qu'il est envisagé sur les auditeurs.

Donnant suite à la question des performances insufflées par le langage, il doit maintenant s'agir de faire la différence entre les performances rituelles, sociales et celles plus proprement théâtrales ${ }^{19}$. Les performances rituelles se basent sur la répétition protocolaire et créent, comme les actes de langage, la réalité sociale en tant que telle. Dans une représentation ou une performance théâtrale, il s'agit de jouer ou de réciter un texte pour lequel la mise en scène se présente sous la condition fictionnelle du «comme $\mathrm{si}^{20} »$. Les performances sociales ont dès lors une relation particulière avec les performances rituelles et théâtrales. Une performance sociale qui a échoué devient une mise en scène prévisible à caractère artificiel, tandis qu'une performance sociale pour ainsi dire plébiscitée produit des effets similaires à ceux du rituel ${ }^{21}$. Ces trois types de performance se distinguent les uns des autres par une configuration spécifique de la répétition, de la citation et de l'effectivité. Non seulement les rituels mais aussi les représentations théâtrales et sociales dépendent de la répétition, même si la différence entre l'ensemble des attentes et l'événement performatif est mise en avant. Finalement, à condition qu'ils n'échouent pas

18. Jacques Derrida, ibid., p. 384-386

19. Une distinction semblable a été proposée par Bernhard Giesen envers qui nous sommes reconnaissant pour ces réflexions, «Performing the sacred. A Durkheimian perspective on the performative turn», dans Jeffrey C. Alexander et al., Social Performance, op. cit., p. 325-367, plus particulièrement p. 338-357. Ce qui importe le plus ici, c'est de conceptualiser et de contextualiser les formes élémentaires d'agir performatif d'une manière systématique.

20. Hans Vaihinger, La philosophie du comme si. Préface et traduction de Christophe Bouriau, Philosophia Scientiae, Cahier spécial, Paris, Kimé, 2008.

21. Jeffrey C. Alexander, «Cultural Pragmatics», op. cit., p. 54. 
face à leurs propres attentes, les rites et les représentations théâtrales définissent une effectivité sociale d'une manière particulière.

Ce dont il est question, c'est bien d'un mécanisme élémentaire du performatif qui informe le trio répétition, citation et effectivité en se rapportant à un principe d'imitation ou plutôt de mimesis. Ce dernier, qui se retrouve par ailleurs déjà chez Platon et Aristote et qui a été travaillé dans les œuvres de Gabriel Tarde et Erich Auerbach, joue un rôle certes considérable dans la formation et la diffusion de l'agir performatif ${ }^{22}$. L'hypothèse de la diffusion et de la stabilisation des modèles performatifs à travers le mécanisme d'imitation n'entraîne en aucun cas une conception statique de l'ordre social cependant. La quasi-impossibilité d'une réitération dans le sens strict du concept a pour conséquence que les performances se différencieront toujours des précédentes en devenant à nouveau sujet d'imitation, et ce, surtout quand elles se singularisent par leur succès et leur effectivité sociale.

Les actes performatifs sont dans un même contexte évolutif de variation, sélection et stabilisation derechef. La différenciation culturelle n'est pas seulement le produit d'un processus évolutif, mais aussi le produit d'actes intentionnels de distinction et d'innovation. C'est ainsi que la réussite de beaucoup de performances sociales dépend de leur individualité et de leur authenticité. Par exemple, personne n'est gêné par le rituel canonisé du mariage même si la déclaration d'amour et la demande en mariage doivent être foncièrement originales et sincères. De la même manière, les performances théâtrales doivent rester originales puisque très peu de spectateurs auraient l'idée d'assister à plusieurs reprises à la même mise en scène d'une pièce de théâtre. La dynamique caractéristique de l'agir performatif devient de ce fait le moteur de la différenciation culturelle. Non seulement les performances font sans cesse l'objet d'imitation, mais elles régénèrent d'autres genres culturels également tels que l'image et la narration. La culture n'est pas uniquement incarnée par des faits matériels ou par des mises en scène performatives, mais mène aussi une existence indépendante dans «l'imaginaire social ${ }^{23} »$ comme arrière-plan culturel d'une société.

Parallèlement à la force pour ainsi dire évolutive de la mimesis, le succès d'une performance, d'une image ou d'une narration dépend directement de

22. Gabriel Tarde, Les lois de l'imitation. Étude sociologique, Paris, Alcan, 1895; Erich Auerbach, Mimésis. La représentation de la réalité dans la littérature occidentale, Paris, Gallimard, 1969. Voir aussi Gunter Gebauer et Christoph Wulf, Jeux, rituels, gestes. Les fondements mimétiques de l'action sociale, Paris, Economica, 2004; Gunter Gebauer et Christoph Wulf, Mimésis. Art-culture-société, Paris, Cerf, 2005 et Christoph Wulf, Zur Genese des Sozialen. Mimesis, Performativität, Ritual, Bielefeld, transcript, 2005, p. 11-83.

23. Cornelius Castoriadis, L'institution imaginaire de la société, Paris, Seuil, 1999; Charles Taylor, «Modern Social Imaginaries», Public Culture, vol. 14, n 1, p. 91-124 de même que Patrick Legros, Frédéric Monneyron, Jean-Bruno Renard et Patrick Tacussel (dir.), Sociologie de l'imaginaire, Paris, Armand Colin, 2006. 
sa résonance dans l'imaginaire social du public - ce qui est une idée qui n'a jusqu'à maintenant pas été assez théorisée ${ }^{24}$. L'emploi métaphorique de la résonance doit clairement démontrer que, dans le domaine de la communication et de la culture, nous n'avons pas affaire à une causalité simple dans l'ordre des interventions. Les signaux reçus et les valeurs perçues font valoir leur influence en faisant vibrer leurs récepteurs, mais seulement conformément aux propriétés de la table d'harmonie culturelle du moment. Les structures latentes culturelles manifestent ainsi leur effet. Et c'est ce qui engendre de nouvelles émotions et significations. Il est alors question d'un double processus: les performances et les autres genres culturels sont, d'une part, alimentés par l'imaginaire social tout en y laissant, d'autre part, leurs propres traces. Tout se passe donc comme si c'était le degré de divergences et de ressemblances entre la performance et l'arrière-plan de modèles culturels déjà existants qui était le principal moteur ou le mécanisme privilégié de l'explication.

\section{La performance rituelle}

D’un point de vue généalogique, le rituel constitue bien le germe de toutes les performances - comme Durkheim l'a montré en discutant l'origine de l'ordre social moderne dans la religion ${ }^{25}$. Le ritualisme a déjà lieu dans le monde animal et dans l'interaction des mères et des nouveau-nés. Or, le rituel au sens propre du terme n'existe que depuis l'apparition des sociétés humaines un tant soit peu complexes. Non seulement le drame occidental remonte aux rituels dionysiaques, mais aussi les compétitions agonales, comme les Jeux olympiques, servaient d'abord à des buts rituels. C'est à partir du rituel que se sont donc développés dans différentes directions aussi bien le théâtre que le sport; ce qui vient nécessiter certaines démarcations conceptuelles. Alors que dans le ritualisme au sens large s'établit une solidarité entre les participants, les représentations théâtrales s'avançant vers la modernité ont eu tendance à instituer une barrière entre le public et la scène en se basant sur des mises en scène fictionnelles. D'une manière semblable, on peut différencier les rituels des jeux agonaux. Pendant la mise en pratique des rituels, en effet, les différences sociales perdent de l'importance, contrairement aux compétitions sportives où l'on part avec des conditions iden-

\footnotetext{
24. Voir Bernhard Giesen, Kollektive Identität. Die Intellektuellen und die Nation 2, Frankfurt/Main, Suhrkamp, 1999; Niklas Luhmann, Ökologische Kommunikation. Kann die moderne Gesellschaft sich auf ökologische Gefährdungen einstellen?, Wiesbaden, VS, 2004; Gerhard Göhler, «Symbolische Politik - symbolische Praxis. Zum Symbolverständnis in der deutschen Politikwissenschaft», dans Barbara Stollberg-Rilinger (dir.), Was heißt Kulturgeschichte des Politischen?, Berlin, Duncker \& Humblot, 2005, p. 57-69.

25. Émile Durkheim, Les formes élémentaires de la vie religieuse, op. cit.
} 
tiques et où l'on se trouve finalement avec des différences, principalement entre le vainqueur et le vaincu ${ }^{26}$.

C'est bien sûr en raison de leur contribution à la question de la solidarité sociétale que la sociologie a prêté une certaine attention aux rituels. Selon Durkheim, la religion ou bien la conscience collective d'une société est «un système solidaire de croyances et de pratiques» qui se rapporte à la distinction entre sacré et profane. Il en ressort que les rituels sont des pratiques collectives qui marquent la différence entre deux ordres cosmiques à ceci près que le sacré n'est rien d'autre qu'une représentation même de la société. À la fois des rituels, comme le sacrifice chez Henri Hubert et Marcel Mauss ${ }^{27}$, sont une médiation entre le sacré et le profane. Il s'agit de voir que les rituels ne sont absolument pas menacés de disparition dans la société moderne, car une position indispensable leur incombe, et ce, en raison de leurs propriétés formelles. Ici, le point de départ de nos réflexions est la définition du rituel de Roy A. Rappaport comme «performance de séquences plus ou moins invariables d'acte formel ou d'énonciation n'étant pas entièrement encodés par les acteurs ${ }^{28} »$. Cette définition met en avant la répétition caractéristique des pratiques rituelles ainsi que la canonisation du savoir rituel ${ }^{29}$. Le déroulement et la signification d'un rituel sont très largement prédéfinis symboliquement et sont, du coup, en grande partie privés de l'interprétation des participants. Dans le mode de l'agir rituel, on fait ainsi beaucoup abstraction de l'intentionnalité individuelle des acteurs ${ }^{30}$. Seule la conformité des participants avec les règles indispensables est décisive pour la réussite d'une performance rituelle. Cette conformité repose sur l'attribution des observateurs, le succès douteux d'un rituel peut ultérieurement encore devenir un sujet de débat. L'anthropologue Stanley J. Tambiah a en outre essayé de faire fructifier le concept de la performativité pour l'analyse des rituels:

L'action ritualisée et ses différentes dimensions est performative dans les trois sens suivants: dans le sens de Austin pour qui dire quelque chose est aussi faire quelque chose en tant qu'acte conventionnel; dans un sens très différent comme performance mise en scène et usant de multiple médiums à travers lesquels les participants font l'expérience intensive de l'événement ; et dans le sens d'une

26. Claude Lévi-Strauss, La pensée sauvage, Paris, Plon, 1962, p. 46.

27. Henri Hubert et Marcel Mauss, «Essai sur la nature et la fonction du sacrifice», L'Année sociologique, vol. 2, 1899, p. 29-138.

28. Roy A. Rappaport, Ritual and Religion in the Making of Humanity, Cambridge, Cambridge University Press, 1999, p. 25.

29. Voir également Stanley J. Tambiah, Culture, Thought and Social Action. An Anthropological Perspective, Cambridge, Harvard University Press, 1985, p. 31 et 128.

30. Caroline Humphrey et James Laidlaw, The Archetypal Actions of Ritual. A Theory of Ritual Illustrated by the Jain Rite of Worship, Oxford, Clarendon Press, 2004. 
valeur indéxicable - je reprend ce concept de Pierce - attachée au, et inférée par les acteurs durant la performance ${ }^{31}$.

Seule la prise en considération de la performativité d'un rituel éclaire ses effets sociaux, son appréhensibilité sensible ainsi que son observation comme symptôme culturel. Primo, le rituel possède en tant que tel, tout comme l'acte de la promesse et de l'excuse, une teneur illocutoire qui le distingue du théâtre ${ }^{32}$. Le rituel accompli mène à un changement du monde social et souvent à une transformation personnelle. Un rituel n'obtient pas d'effet de réalité par une ingérence directe et physique sur son environnement, mais par ses propriétés formelles et de convention sociale ${ }^{33}$. Secundo, une performance rituelle est vécue physiquement et sensuellement, ce qui n'est absolument pas insignifiant pour sa signification et son effet $^{34}$. Tertio, et non des moindres points, un rituel peut être lu comme un indice ou un symptôme culturel qui fait référence à des imaginaires sociaux partagés - pensons ici au combat de coqs balinais comme «commentaire metasocial ${ }^{35}$ ». Les imaginations collectives de pureté et d'impureté, de masculinité et de féminité trouvent par exemple dans les rituels non seulement une expression physique, mais sont également consolidées symboliquement.

Pour complexe que cela puisse paraitre, c'est en se fondant sur la force transformative des performances rituelles qu'il devient possible de démontrer comment les qualités performatives d'un rituel sont associées à ses propres propriétés formelles. C'est que l'exécution d'un rite est fondée sur une forme particulière de règle: pendant qu'il est question de normes sociales comme règles qui régulent sous forme d'attentes partagées un comportement déjà existant, ces mêmes règles constitutives d'un rituel fixent ce qui a cours comme un rituel précisément ${ }^{36}$. Pour dire la même chose autrement, et de manière presque tautologique, les instructions rituelles constituent la mise en pratique d'un rituel de la même façon que les règles du jeu constituent le jeu. Les règles constitutives d'un jeu d'échecs ou du rituel de la communion constituent la pratique respective comme telle, alors que les règles régulatrices de la circulation routière, par exemple, structurent une pratique déjà

31. Stanley J. Tambiah, Culture, Thought and Social Action, op. cit., p.128.

32. Ibid., p. 134.

33. Voir Roy A. Rappaport, Ritual and Religion in the Making of Humanity, op. cit., p. 46-50.

34. Voir Bernhard Giesen, «Performing the sacred», op. cit., p. 342 et Lawrence E. Sullivan, «Sound and Senses. Towards a Hermeneutics of Performance», History of Religions, vol. 26, n 1, 1986, p. 1-33.

35. Clifford Geertz, The Interpretation of Cultures. Selected Essays, New York, Basic Books, 2006, p. 448. Selon Catherine Bell, il incombe à presque toutes les théories du rituel que ce dernier surmonte la dichotomie de la pensée et de l'agir, voir Ritual Theory, Ritual Practice, Oxford, Oxford University Press, 1992. Le rituel est à classer avant tout dans le domaine de l'agir par lequel se développe la dichotomie. Bell voudrait donc que cette dichotomie soit surmontée dans la notion du rituel vue comme une pratique, tandis que l'approche proposée ici veut conserver la distinction entre la pratique rituelle et l'imaginaire social et la faire fructifier.

36. Voir John Rawls, «Two Concepts of Rules», The Philosophical Review, vol. 64, n 1, 1995, p. 3-32 et John R. Searle, The Construction of Social Reality, Londres, Lane, 1995, p. 43. 
existante. À cet égard, les rituels ressemblent dans leur structure à un acte de langage qui crée la réalité sociale ${ }^{37}$. Cependant, une nette séparation entre les règles constitutives et régulatrices n'est pas toujours possible. Si le signe de croix avec de l'eau bénite en tant que vestige de l'ablution rituelle opère encore sur le croyant la transformation purificatoire ou bien si la norme sociale règle uniquement l'entrée dans une église catholique, cela dépend tout d'abord de l'expérience du participant et ensuite de l'encadrement par l'Église comme institution sociale.

Le rituel est encore fondé sur cette importante règle constitutive que Victor Turner et Arnold van Gennep nomment la liminalitê38. L'emploi de règles constitutives est accompagné d'un franchissement de seuil qui permet la transformation de personnes et de situations. Dans la phase liminale du rituel, la validité des normes quotidiennes est annulée, une caractéristique que Turner qualifie de contre-structure («antistructure»). La contre-structure d'un rituel démontre en outre que - pour la durée de ce dernier - les différences sociales entre les participants, les différences de caste ou de statut par exemple, sont reléguées au second plan. Ainsi les rituels provoquent un sentiment extraordinaire de communauté, quelque chose comme une pure communitas $^{39}$ qui doit donc rester étranger à la vie quotidienne avec ses différents rôles sociaux. C'est justement parce que les rituels annulent pour un certain temps la structure sociale qu'ils contribuent à l'ordre social, c'est-àdire en transformant la communitas éphémère en solidarité communautaire. Les rituels constituent ainsi un mécanisme éprouvé pour maîtriser les crises, comme le montre non seulement la danse de la pluie des indiens hopis ${ }^{40}$ mais aussi le travail collectif de deuil après le 11 septembre $2001^{41}$. Après la phase de seuil et le retour dans la société, le statut social d'une personne peut faire l'objet de changements durables, comme c'est le cas dans les initiations et les investitures. Parallèlement, même les sociétés les plus modernes ont toujours recours aux formes rituelles lors d'un changement important. À terme, c'est

37. Voir Bernhard Giesen, «Performing the sacred», op. cit., p. 340; Pierre Bourdieu, Ce que parler veut dire, op. cit., p. 103-134.

38. Arnold Van Gennep a entre autres analysé dans son étude classique sur les rituels de transition toute la question des rites de passage, voir Les rites de passage, Paris, É. Nourry, 1909. II a constaté qu'un rite de transition se subdivise en trois phases: d'abord se déroule la séparation de la collectivité, ensuite vient la phase de seuil ou phase liminale et enfin la réinsertion dans la communauté. Ce modèle a été étendu par Victor W. Turner à l'ensemble des rituels et sur les autres genres culturels, The Ritual Process. Structure and Anti-Structure, New Brunswick, Aldine Transaction, 2009.

39. Victor W. Turner, The Ritual Process, op. cit., p. 96 et p. 131-165.

40. Robert K. Merton, «Manifest and Latent Functions. Toward the Codification of Functional Analysis in Sociology», dans On Theoretical Sociology. Five Essays Old and New, New York, Free Press, 1967, p. 73-138, plus particulièrement p. 118-119.

41. Voir entre autres Edward A. Tiryakian, «Durkheim, solidarity, and September 11 », dans Jeffrey C. Alexander et Philip Smith (dir.), The Cambridge Companion to Durkheim, Cambridge, Cambridge University Press, 2005, p. 305-321. 
ainsi autant l'obligation transindividuelle des règles rituelles que le sentiment de communauté qui font du rituel une véritable affaire collective, un véritable culte commun.

Au risque de se répéter, la sociologie des religions de Durkheim fut dans la réception sociologique de la notion du rituel de la plus grande importance. Pour toute la microsociologie, ses réflexions sur le culte de l'individu furent influentes ${ }^{42}$. Selon Erving Goffman par exemple, la sainteté de la personne dans la société moderne se manifeste par des techniques pour «sauver la face», pour «manager l'impression» ainsi qu'à travers des rituels de déférence ${ }^{43}$. Autre exemple, c'est bien à la suite de Durkheim et de Goffman que Randall Collins a fait du rite d'interaction la référence de base de sa microsociologie $^{44}$. Pour lui, non seulement les hommes et les sociétés, mais aussi les actes privés, comme la pensée, sont décrits en tant que chaînes de rites d'interaction. Pour la macrosociologie cette fois, la notion du rite de Durkheim fut appliquée surtout dans des ouvrages concernant les mass media et les publics ${ }^{45}$. Les publications d'Edward Shils sur le centre sacral des sociétés modernes, et en particulier sur son analyse du couronnement de la reine d'Angleterre menée avec Michael Young, furent certainement innovatrices à cet égard ${ }^{46}$. Les travaux sur les événements médiatiques de Daniel Dayan et Elihu Katz ainsi que l'analyse du scandale du Watergate de Jeffrey C. Alexander poursuivent un objectif similaire ${ }^{47}$. Alexander a développé son approche du rituel en une théorie de la performance sociale qui, comme la théorie des chaînes des rites d'interaction de Collins, vise à lier l'agir microsocial et les structures macrosociales - il s'agira d'y revenir.

\section{La performance théâtrale}

Dans l'état des connaissances actuelles, le théâtre occidental est issu des rituels grecs de Dionysos $^{48}$. Cela dit, et malgré leur origine dans un rituel religieux, les performances théâtrales se distinguent des performances rituelles par la mise en place du «comme $\mathrm{si}^{49}{ }^{»}$. Au moment où un agir est cadré en tant que

42. Émile Durkheim, De la division du travail social, Paris, Les Presses Universitaires de France, 1967, p. 157; Émile Durkheim, «L'individualisme et les intellectuels», Revue Bleue, vol. 10, n 1, 1898, p. 7-13.

43. Erving Goffman, Interaction Ritual. Essays in Face-to-Face Behavior, New Brunswick, Aldine Transaction, 2005.

44. Randall Collins, Interaction Ritual Chains, Princeton, Princeton University Press, 2004.

45. Une vue d'ensemble se trouve chez Simon Cottle, "Mediatized rituals: beyond manufacturing consent», Media, Culture \&s Society, vol. 28, n³, 2006, p. 411-32.

46. Edward Shils et Michael Young, "The Meaning of the Coronation», dans Edward Shils, Center and Periphery. Esays in Macrosociology, Chicago, University of Chicago Press, 1975, p. 135-152.

47. Daniel Dayan et Elihu Katz, "Articulating Consensus. The Ritual and the Rhetoric of Media Events», dans Jeffrey C. Alexander (dir.), Durkheimian Sociology. Cultural Studies, Cambridge, Cambridge University Press, 1988, p. 161-186; Jeffrey C. Alexander, «Watergate as Democratic Ritual», op. cit.

48. Voir Roland Barthes, L'obvie et l'obtus. Essais critiques III, Paris, Seuil, 1982, p. 63-85.

49. Hans Vaihinger, La philosophie du comme si, op. cit. 
performance théâtrale, celui-ci n'est plus imputé à la personne de l'interprète, mais au personnage qui est interprété. L'agir interprété se caractérise dès lors par sa très haute fictionalité. De plus, la performance théâtrale implique une différence entre les acteurs et l'audience qui s'est graduellement imposée dans le passage, par exemple, du théâtre antique au théâtre bourgeois. Les représentations théâtrales dites avant-gardistes essaient de franchir encore et toujours cette frontière, mais en l'occurrence elles la reproduisent à nouveau. Si ce n'était pas le cas, les différences constitutives pour le théâtre disparầtraient et la représentation théâtrale ne serait plus reconnaissable.

La transformation de la personne n'est pas un résultat indispensable des performances théâtrales. C'est que le théâtre ne doit pas forcément instruire, mais qu'il peut tout simplement distraire sans pour autant par là altérer quoi que ce soit de son essence. Et quand, en effet, une transformation doit avoir lieu, elle s'effectue du côté du public et non pas de celui des acteurs. L'antique théorie du drame d'Aristote est toujours très proche du rituel, ce qui peut être démontré entre autres par le fait que la tragédie doit purifier le spectateur de ses passions par la compassion (catharsis). Une moralisation du spectateur, Friedrich Schiller l'avait en tête quand il a présenté la scène théâtrale comme un établissement moral («die Schaubühne als moralische Anstalt») au service rationaliste d'une éducation esthétique du genre humain ${ }^{50}$. L'art moderne, qui a pris de plus en plus conscience de son autonomie, a renoncé à ces fonctions rituelles et pédagogiques et laisse ainsi libre l'effet transformatif au public. Si l'art moderne a encore une fin en dehors de luimême, ce serait vraisemblablement un mur de projection et un objet de réflexion. Du fait que les performances théâtrales donnent une représentation fictionnelle sur la scène, elles permettent au public de réfléchir sur lui-même. Il y a eu depuis Artaud encore et toujours des efforts faits pour que le théâtre se rapproche du rituel et pour intégrer plus fortement le spectateur. Le «Performance Art» contemporain constitue aussi à cet égard un cas limite de performance théâtrale, parce qu'il conteste la répétitivité et la fictionalité de ce type de performance à cause de son événementialité et de sa corporalité ${ }^{51}$. Souvent l'art performatif suspend la différence entre l'artiste et le spectateur, que ce soit la représentation qui se déroule dans un espace public sans la référence explicite à sa théâtralité, ou que ce soit le spectateur qui est forcé d'intervenir par la représentation.

C'est donc le propre de la fictionalité qui se retrouve au centre de la performance théâtrale. Or voilà, comme l'a fait remarquer Wolfgang Iser, la

50. Friedrich Schiller «Was kann eine gute stehende Schaubühne eigentlich wirken?», Theoretische Schriften, Werke und Briefe, vol. 8, Frankfurt/Main, Deutscher Klassiker-Verlag, 1992, p. 185-200.

51. Voir Bernhard Giesen, «Performance Art», dans Jeffrey C. Alexander et al., Social Performance, op. cit., p. 315-324. 
fictionalité se comprend comme un principe intermédiaire à la fois subtil et complexe transmis entre le réel et l'imaginaire ${ }^{52}$. Seul l'acte de feindre, dit Iser, rend l'imaginaire réel ou le rituel présent. Une performance au sens théâtre s'appuie sur la corporéité et la présence physique, et ce, tout en ayant recours aux motifs narratifs et aux archétypes répandus dans la société. Les films et les séries télévisées fictionnels n'y échappent pas puisqu'ils sont également des performances théâtrales - par le biais de la médiation fournie par les supports d'enregistrement et de lecture. Ce sont ainsi tous les genres de fiction qui représentent toujours une imitation du réel. Même si les personnages sont inventés, des éléments de la représentation peuvent tout à fait être réalistes. Face à une scène de torture, nous pouvons nous demander si la torture se déroule vraiment ainsi ou si les cris du torturé étaient assez près de la réalité. Même un genre comme la science-fiction ne peut se passer d'éléments familiers en sorte que ce soit bien toute performance-représentation qui peut devenir à la fois l'objet d'imitation et le centre d'attention de la critique médiatique ${ }^{53}$.

La conception du monde comme scène et celle de la société comme théâtre est presque aussi ancienne que le théâtre occidental lui-même. Ainsi Platon conceptualise dans son nomoi l'être humain comme étant une marionnette des dieux (644d-645b) et éconduit les poètes tragédiens avec la justification que le véritable État est lui-même un drame, qui se voue à l'imitation de la plus belle et de la meilleure vie (817a-d). La métaphore du théâtre a également exercé une fascination durable sur la sociologie moderne, ce que démontre très bien la carrière du concept de rôle. Pour Mead, par exemple, "prendre le rôle les uns des autres» est certainement plus qu'une simple métaphore puisqu'elle devient un principe élémentaire d'un social déjà visible dans la pratique enfantine du jeu de rôles ${ }^{54}$. Goffman a également essayé de défricher le vaste champ des interactions sociales avec l'aide des catégories théâtrales comme la scène, le rôle, le script et l'ensemble ${ }^{55}$. La fertilité de ces

52. Wolfgang Iser, Das Fiktive und das Imaginäre. Perspektiven literarischer Anthropologie, Frankfurt/Main, Suhrkamp, 1991.

53. Ainsi la série télévisée 24 heures chrono a été au centre de la critique après le scandale des photos d'Abu Ghraib glorifiant la torture. Surtout l'article de Jane Mayers dans The New Yorker ( «Whatever it takes. The politics of the man behind "24" ") a enflammé le débat parce qu'elle y a relaté que les membres de l'armée américaine se sont plaints auprès du bureau de production que la série influençait l'attitude des cadets par rapport à la torture et que le héros torturé de la série, Jack Bauer, a été perçu comme un idéal par les soldats dans les régions en guerre. Que la performance théâtrale de «torturer/ savior fusion» dans 24 heures chrono se soit heurtée à tant de résonance dans la société américaine est, d'une part, due aux attentats du 11 septembre et, d'autre part, au motif du «law defying hero » très répandu dans la culture populaire américaine; voir Stephen Holmes, «Is Defiance of Law a Proof of Success? Magical Thinking in the War on Terror», dans Karen G. Greenberg (dir.), The torture debate in America, New York, Cambridge University Press, p. 118-135.

54. George H. Mead, Mind, Self, and Society. From the Standpoint of a Social Behaviorist, Chicago, University of Chicago Press, 1970.

55. Erving Goffman, La mise en scène de la vie quotidienne, op. cit. 
approches suggère ainsi de ne pas seulement attribuer au théâtre une valeur métaphorique et heuristique pour la sociologie, mais de partir du principe que la culture humaine même est dramatologique ${ }^{56}$.

Cette conjoncture du théâtre dans la sociologie doit être vue dans son contexte sociétal et donc dans la multiplication des contextes d'agir. L'intersection des cercles sociaux ${ }^{57}$ et la différenciation fonctionnelle de la société moderne ${ }^{58}$ conduisent à ce que les acteurs ne soient plus inclus que comme des porteurs de rôles dans des contextes institutionnels. Goffman a en outre démontré que même le vrai soi n'est rien d'autre qu'un rôle qui, par conséquent, dépend de sa projection, de sa présentation ${ }^{59}$. C'est la personne qui devient elle-même un masque théâtral. Cela étant dit, la contemplation du monde comme étant une scène se heurte à certaines limites. Souvent, les performances quotidiennes aspirent à garder pour elles-mêmes leurs propres caractères artificiels et fictionnels, souvent aussi à les cacher à l'acteur luimême - sauf peut-être à ce qu'une conscience de la distance émerge et qu'à ce moment l'auto-mise en scène soit rendue expressément reconnaissable et ironisée.

Tel que nous l'avons discuté ci-haut, si les performances rituelles font naître une «communitas", les performances théâtrales créent plutôt un espace public par la séparation des interprètes et de l'auditoire - et c'est ce dernier concept qui doit du coup être problématisé. À la performance théâtrale correspond le modèle d'un auditoire courtois ou représentatif. Et c'est celui-ci qui va devenir un "public» dans la modernité; ce dont discute par ailleurs Habermas dans Strukturwandel der Öffentlichkeit ${ }^{60}$. Avec le public civil, c'est un naturel factice favorisant le type de l'agir de la performance sociale qui entre en jeu en remplaçant la sophistication de la Cour. Chez Habermas, c'est la narration héroïque des débuts pleins d'espoir du public civil qui bascule sous la pression d'une industrie culturelle défigurante et d'un public produit par les mass média dans une histoire de déclin. Le travail présent rejette l'idée d'un domaine public civil qui serait, comme par magie, quelque chose de profondément naturel à l'origine. Au lieu de cela, il doit s'agir de souligner que le naturel et le consensus sont toujours artificiels, c'est-à-dire performatifs construits et fabriqués.

56. Wolfgang Lipp, «Kultur, dramatologisch», Österreichische Zeitschrift für Soziologie, vol. 9, n 1, p. 8-25.

57. Georg Simmel, Soziologie. Untersuchungen über die Formen der Vergesellschaftung, vol. 11, Simmel, Georg: Gesamtausgabe, Frankfurt/Main, Suhrkamp, 1992 [1908], p. 456-511.

58. Niklas Luhmann, «Differentiation of Society», The Canadian Journal of Sociology/Cahiers canadiens de sociologie, vol. 2, n 1, 1977, p. 29-53.

59. Erving Goffman, La mise en scène de la vie quotidienne, op. cit.

60. Jürgen Habermas, Strukturwandel der Öffentlichkeit. Untersuchungen zu einer Kategorie der bürgerlichen Gesellschaft, Frankfurt/Main, Suhrkamp, 1990. 


\section{La performance sociale}

Nos sociétés contemporaines se caractérisent par la différenciation socioculturelle et la contrainte à l'individualisation. Il se crée ainsi des marges de manœuvre qui ne peuvent pas toujours être remplies par des rituels standardisés. Plus encore, et pour paradoxal que cela puisse paraitre, c'est le processus de socialisation (Vergesellschaftung) et de différenciation des rôles qui mène à un besoin croissant de communauté et d'authenticité. C'est ce qui se voit, par exemple, dans la réaction politique aux catastrophes et aux crises alors qu'il s'agit d'éviter de donner l'impression qu'un rituel du regret et de la désolation se répète simplement, sans trop y croire ou en y jouant seulement son rôle. La structure moderne des rôles et, à plus forte raison, le jeu des rôles postmoderne mène à un malaise du modernisme se traduisant lui-même par la recherche de l'authenticité perdue ${ }^{61}$. Cela se manifeste entre autres dans la fragmentation des milieux sociaux et des sous-cultures et dans le retour du fondamentalisme religieux. C'est donc le caractère arbitraire du postmoderne qui favorise les nostalgies de l'authenticité dont la satisfaction temporaire est promise par un troisième type de performance - la performance sociale.

La transition du rituel au théâtre s'est accomplie dans l'Antiquité grecque moyennant une étape intermédiaire qui est d'emblée performative. Le dithyrambe est une improvisation lyrique individuelle qui, en tant que partie du culte classique de Dionysos, est représenté en alternance avec le reste de la communauté culturelle comme chœur. Ce qui est intéressant ici, c'est que le dithyrambe donne à penser un modèle ou une structure qui est déjà non loin des performances sociales et du public moderne. D'un côté, la performance lyrique pouvait plus ou moins bien réussir; de l'autre, l'alternance entre l'improvisateur et le public participant est bien l'ancêtre de la division dramatique entre les interprètes et le public. Au sein du public politique d'aujourd'hui, entre autres exemples, ce sont bien les mass media et le journalisme qui remplacent le chœur en mettant en scène un dialogue entre "performeurs» et public par des méthodes et des techniques appropriées (commentaires, vox pop, lettres de lecteurs, interviews, etc.). La place du public théâtral y est prise par un public plus ou moins participant, dont les membres peuvent en principe eux-mêmes se produire à tout moment comme les «performeurs». Les acteurs politiques, quant à eux, se servent de ces mêmes techniques, en se mettant perpétuellement en scène, que ce soit

61. Voir Peter L. Berger, «On the obsolecence of the concept of honor», European Journal of Sociology, vol. 11, n², p. 338-347; Helmuth Plessner, Grenzen der Gemeinschaft. Eine Kritik des sozialen Radikalismus, Frankfurt/Main, Suhrkamp, 2002 et Charles Taylor, A Secular Age, Cambridge, Harvard University Press, 2007, p. 473-504. 
dans la rue lors d'une discussion entre citoyens ou en s'entourant d'un choix représentatif de l'électorat lors de rassemblements.

La conception de la performance sociale qui est utilisée ici est surtout issue de la pragmatique culturelle de Jeffrey C. Alexander ${ }^{62}$. Bien entendu, les autres formes de performance sont sociales, ce pourquoi cette notion donne facilement l'impression d'une infélicité terminologique. Même si le concept de la performance sociale parait à première vue tautologique, il désigne une forme spécifique de performance. Alors que la pragmatique linguistique s'occupe des effets des actes de langage sur les auditeurs, Alexander s'intéresse au succès relatif d'une performance sociale en rapport avec l'arrière-plan culturel du public - les dites «background representations» que l'auteur emprunte ouvertement à la théorie durkheimienne du rituel. $\mathrm{Si}$, dans les sociétés dites simples, un savoir canonique et un arrière-plan culturel commun sont peu problématiques, cette hypothèse devient tendanciellement de plus en plus douteuse en avançant vers les sociétés modernes ${ }^{63}$. Une performance sociale réussie doit fusionner avec l'horizon culturel du public si elle veut remplir une fonction rituelle. Ainsi, le succès de la communication publique peut être compris comme étant le résultat de performances contingentes. Alors que la conformité représente la condition requise pour un rituel réussi, il est d'abord question d'authenticité pour le succès des performances sociales ${ }^{64}$. Non seulement la prétention de véracité, mais aussi la vérité des assertions et la valeur normative doivent être performativement rehaussées ${ }^{65}$.

Les performances sociales, surtout celles du monde de la politique, se doivent de citer des scripts culturels et imaginaires qui soient proprement populaires si elles veulent conquérir leur public. Ainsi Charles de Gaulle lors de sa visite au Québec en 1967 - à bord d'un bateau remontant le SaintLaurent - a cité l'explorateur français Jacques Cartier. En mai 2003, lors d'un discours sur la fin officielle de l'opération de combat en Irak, George W. Bush a pris le rôle de Tom Cruise dans Top-Gun et s'est mis en scène sur le porte-avions Abraham Lincoln comme le premier soldat de l'État. De manière similaire, la génuflexion de Willy Brandt devant le ghetto de Varsovie a pu déployer son effet surtout, sinon seulement comme «imita-

62. Jeffrey C. Alexander, «Cultural Pragmatics...», op. cit.; pour le commentaire, voir aussi Jonathan Roberge, «Jeffrey C. Alexander et les dix ans du programme fort en sociologie culturelle», Cahiers de recherche sociologique, $n^{\circ} 47$, janvier 2009, p. 47-66, plus particulièrement p. 54-59.

63. La comparaison d'Alexander d'un arrière-plan culturel homogène («fused») dans les sociétés simples et d'un arrière-plan culturel fragmenté ( «defused») dans les sociétés modernes doit être traitée avec précaution.

64. Voir aussi Bernhard Giesen, «Performing the Sacred», op. cit., p. 350-357.

65. Nous n'avons qu'à nous rappeler le destin du chercheur d'étoiles orientales du Petit Prince d'Antoine de SaintExupéry. On a seulement prêté attention et foi à sa découverte après qu'il a adapté sa présentation aux habitudes scolaires en Occident. 
tio Christi ${ }^{66} »$. Dernier exemple, la littérature scientifique sur la performance s'est aussi employée à la description des attentats terroristes comme étant précisément organisés pour un public ${ }^{67}$ et visant un effet de $\operatorname{choc}^{68}$. Ironiquement, non seulement les accessoires de la civilisation occidentale (par exemple les avions) ont été utilisés pour l'attentat terroriste sur les tours jumelles du World Trade Center, mais les actes de terreur trouvaient un fort écho dans la culture occidentale et dans les fantaisies de destruction issues de la culture populaire de Fight Club à Independence Day.

Geoffrey Hartman révèle une autre dimension performative du terrorisme en attribuant au fondamentalisme moderne une "quête d'authenticité $^{69} »$. En regardant de plus près, le message passé à travers l'acte de terreur ne s'adresse pas seulement au public, mais au «performeur» lui-même qui, en sacrifiant sa propre vie, s'assure d'une façon performative de l'authenticité de sa foi. Le terrorisme motivé du fondamentalisme a alors peut-être plus à voir avec la production d'une authenticité performative qu'avec des exigences politiques concrètes ou une résistance diffuse contre l'impérialisme.

Par l'entremise des mass media modernes, l'agir performatif de chacun peut donc au moins potentiellement avoir des effets sur toute la société, comme le montre le phénomène du terrorisme. Les performances sociales comme événements médiatiques annulent la dichotomie entre «micro» et « acro $^{70} »$. Pour reprendre cet exemple, les politiciens essaient par des performances authentiques de créer le charisme qui, au-delà du traditionalisme et de la rationalité, influence les décisions d'un électorat volatile. Des actes performatifs isolés peuvent toujours ainsi devenir le déclencheur de développements sociaux en rencontrant une certaine résonance dans la société.

\section{Les drames sociaux et les drames fictionnels}

Il reste encore à considérer deux processus sociaux qui constituent des formes élémentaires d'agir performatif: les drames sociaux en société (social drama) et les drames fictionnels mis en scène (stage drama) sur lesquels notamment Victor Turner et Richard Schechner se sont penchés. Un drame

66. Valentin Rauer, «Willy Brandt's Kneefall at the Warsaw Memorial», dans Jeffrey C. Alexander et al., Social Performance, op. cit., p. 257-282; Christoph Schneider, Der Warschauer Kniefall. Ritual, Ereignis und Erzählung, Konstanz, UVK, 2006.

67. Bernhard Giesen, Zwischenlagen. Das Außerordentliche als Grund der sozialen Wirklichkeit, Weilerswist, Velbrück, 2010, p. 212; Richard Schechner, «9/11 as Avant-Garde Art?», PMLA, 2009, p. 1820-1829.

68. Jeffrey C. Alexander, "From the Depths of Despair. Performance, Counterperformance, and "September 11" ", Sociological Theory, vol. 22, $n^{\circ}$ 1, 2004, p. 88-105.

69. Geoffrey H. Hartman, Scars of the Spirit. The Struggle against Inauthenticity, New York, Palgrave Macmillan, 2002, p. 233.

70. Jeffrey C. Alexander, Bernhard Giesen, Neil Smelser et Richard Münch (dir.), The Micro-Macro Link, Berkeley, University of California Press, 1987. 
social, au contraire du rituel et de la tragédie classique, possède un dénouement qui ne peut se deviner. Il peut être divisé suivant quatre actes consécutifs $^{71}$ : la rupture (breach) représente une violation publique de la norme qui s'étend à la crise (crisis) et, par voie de conséquence, à un conflit entre partis divers; le redressement (redress) renvoie à l'effort consistant à surmonter la crise sociale. Le dernier acte du drame social a lui-même deux dénouements possibles: la reconnaissance du schisme (recognition of the schism) ou l'intégration (reintegration).

Les drames sociaux se forment surtout à la faveur de conflits externes ou bien à la suite de tensions latentes à l'intérieur d'un groupe se manifestant alors à travers la dramatisation publique. Sans les tensions latentes de la Troisième République et le conflit avec l'Allemagne, l'affaire Dreyfus n'aurait certainement jamais dépassé les limites d'un cas d'espionnage. Dans chaque acte du drame social, les performances rituelles et sociales jouent ainsi un rôle important. La rupture peut être mise en scène d'une façon dramatique, comme pendant le Boston Tea Party, ou elle peut être rendue publique à travers un acte performatif de révélation, comme Zola a réussi avec son «J'accuse». Cela étant dit, le rituel et la performativité sont aussi ce par quoi l'intégration peut se re-présenter. Pour surmonter la crise, en effet, ce sont surtout l'action et l'incarnation d'un certain symbolisme qui réconcilient les différences sociales et recréent de la solidarité.

La nomination rituelle du président américain, pour prendre cet exemple, met fin à une campagne électorale qui déchire le peuple en sorte qu'elle incarne un tel mécanisme de dépassement ${ }^{72}$. Une autre possibilité classique est le sacrifice rituel d'un bouc émissaire, qui se tient en dehors des partis se disputant et qui permet ainsi de surmonter la rupture de la communautét ${ }^{73}$. Même l'ouverture de la procédure de destitution à l'encontre de Nixon relève d'un mécanisme semblable puisque c'est son autosacrifice forcé qui a conduit à l'achèvement du scandale du Watergate. Autrement dit, il faut très souvent qu'une exclusion symbolique des coupables s'ensuive pour qu'un conflit soit réglé et que la crise sociale soit surmontée. Ce qui est justement très loin d'être évident. Le droit, en outre, est une autre manière de dépasser la crise, mais comme l'affaire Dreyfus l'a notamment montré, cette tentative performative peut tout à fait échouer si elle se heurte à la résonance et à l'acceptation dans toute la société. Intégration ou reconnaissance du schisme alors? Ce qu'il faut surtout comprendre, c'est que la réponse à cette

71. Victor W. Turner, From Ritual to Theatre. The Human Seriousness of Play, New York, PAJ, 2004, p. 69-71.

72. Voir Christoph Wulf, Zur Genese des Sozialen, op. cit., p. 153-163.

73. Voir René Girard, La violence et le sacré, Paris, Hachette Littératures, 2008. 
question est le résultat de performances à la fois contingentes et lourdes de conséquences.

Schechner a porté son attention sur le fait que les performances théâtrales et autres drames fictionnels font sans cesse des emprunts à des drames sociaux qui, eux-mêmes, recourent constamment aux scripts et motifs des textes et des interprétations fictionnels. Pour le dire succinctement, les uns et les autres se réfèrent aux uns et aux autres à travers des actes d'imitation productive (mimesis). Turner l'illustre très bien par l'entremise de l'exemple d'un scandale du Watergate qui se déroula presque "prêt pour la scène » :

Le drame social du Watergate était plein de «stage business» durant toutes ses phases, de l'atmosphère de conspiration de l'épisode de «breach» signalé par la découverte de l'enregistrement incriminant, à travers la fictionalité limpide du camouflage de l'affaire et jusqu'à la phase de crise propre à l'investigation avec les révélations de Gorge profonde et la combinaison de principes nobles et d'opportunisme. La phase de récupération («redressive phase») n’était pas moins mise en scène à travers des modèles à la fois théâtraux et fictifs ${ }^{74}$.

Et ce n'est pas tout puisque le Watergate fut lui-même objet d'imitation pour les performances théâtrales sur scène et à la télévision. Ainsi se referme le cycle mimétique de la culture qui ne s'oppose pas à la transformation sociale, mais la fait plutôt avancer.

\section{Conclusion}

En résumant, on peut dire que la métaphore du théâtre enrichit aujourd'hui la recherche sociologique, ce qui se voit entre autres dans la conjoncture récente autour de l'idée de la performativité. La typologie systématique de l'agir performatif présentée revendique la possibilité d'une application empirique, mais n'est en aucun cas sans alternative. Par exemple, d'aucuns pourraient aussi introduire le jeu libre et la compétition sportive, respectivement des performances ludiques et agonales, comme étant des catégories à la fois autonomes et essentielles. À ces deux formes manque toutefois - dans l'interprétation défendue ici - la nécessité d'une représentation scénique comme elle est déjà donnée dans le rituel simple. Le concept répandu de «performance culturelle ${ }^{75}$ » dans l'anthropologie n'est certes pas soumis à cette objection, pourtant il ne déploie pas les différences importantes à l'intérieur de la catégorie de l'agir performatif. Au demeurant, la classification ici proposée entre performances rituelle, théâtrale et sociale est idéal-typique

\footnotetext{
74. Victor W. Turner, From Ritual to Theatre, op. cit., p. 74.

75. Voir par exemple Milton B. Singer, Traditional India. Structure and Change, Philadelphie, American Folklore Society, 1959 de même que John J. MacAloon, Rite, Drama, Festival, Spectacle. Rehearsals Toward a Theory of Cultural Performance, Philadelphie, Institute for the Study of Human Issues, 1984.
} 
et a surtout pour but de dresser un tableau des différentes possibilités. Les transitions empiriques sont bien sûr floues, comme l'ont montré entre autres les multiples va-et-vient entre le théâtre moderne et le rituel. Il s'agirait donc de regrouper les résultats dans le tableau suivant:

\begin{tabular}{|l|l|l|l|}
\hline \multicolumn{1}{|c|}{ Performance } & \multicolumn{1}{c|}{ Rituel } & \multicolumn{1}{c|}{ Théâtre } & \multicolumn{1}{c|}{ Performance sociale } \\
\hline Condition de réussite & Conformité & Fictionalité & Authenticité \\
\hline Effets sociaux & $\begin{array}{l}\text { Solidarité et } \\
\text { Transformation }\end{array}$ & $\begin{array}{l}\text { Divertissement } \\
\text { et Réflexion }\end{array}$ & Résonance et fusion \\
\hline Différenciation & Communauté & interprète/audience & «Performeur»/public \\
\hline
\end{tabular}

Avec la différenciation croissante de la société, une diversification des actes performatifs se produit. Le théâtre et la performance sociale ne prennent jamais exactement la place du rituel, mais l'accompagnent de manière subtile et complexe. Les obligations nées du rituel restent au centre de la coordination des interactions sociales, de la fondation des institutions et du règlement des conflits sociaux. Les performances théâtrales, quant à elles, créent un contre-monde fictif qui ne sert pas seulement au divertissement et à la réflexion, mais qui proposent des modèles pour imitation dans la vie quotidienne. Dans la vie sociale, la théâtralité exhibée montre qu'on ne s'absorbe pas dans son rôle social, et elle apporte ainsi une contribution à la protection de la personnalité. Qui joue avec son rôle s'émancipe de celui-ci et crée ainsi une sphère privée dans laquelle la dignité de l'individu reste protégée. Ce qui ne manque jamais d'être surprenant, c'est ainsi de voir que les performances sociales deviennent indispensables au fur et à mesure que l'individualisation et l'authenticité en tant que valeurs sociales triomphent, que les obligations traditionnelles et l'homogénéité culturelle d'une société disparaissent et que le public devient l'instance centrale de médiation pour la vie en société. L'unité culturelle des différentes performances est ainsi établie, d'un côté, par des rapports réciproques comme autant, de l'autre, par un travail incessant sur l'imaginaire collectif. Les récits comme les représentations narratives des actes performatifs peuvent faire objet d'imitation et ainsi devenir une référence pour d'autres actions. Naturellement, cela s'applique aussi aux images d'après lesquelles nous formons nos corps de même qu'aux modèles qui nous livrent des directives pour notre propre manière de vivre. Les actes performatifs constituent non seulement une intersection entre les actions au sens strict et ces formes culturelles, mais partagent aussi quelquesunes de leurs propriétés avec les narrations et les images. Cette idée donc de performativité promet énormément et, entre autres, de rendre explicable la 
relation entre l'agir et la culture au niveau théorique et empirique. Mais pour que cela se réalise parfaitement, encore beaucoup de travaux conceptuels et d'études empiriques seront nécessaires.

Traduit de l'allemand par Sophie Schinko, Werner Binder et Francis Le Maître 